\title{
Preparing young adults with disability for employment
}

\author{
Brian J. Taylor ${ }^{1}$ BSc CEd cosw, Sinéad McGilloway ${ }^{2} \mathrm{PhD}$ and Michael Donnelly ${ }^{1} \mathrm{PhD}$ \\ ${ }^{1}$ Department of Epidemiology and Public Health, Queen's University Belfast, Northern Ireland and ${ }^{2}$ Department of \\ Psychology, National University of Ireland at Maynooth, Ireland
}

\author{
Correspondence \\ Sinéad McGilloway \\ Department of Psychology \\ National University of Ireland \\ at Maynooth \\ Maynooth \\ County Kildare \\ Ireland \\ E-mail: sinead.mcgilloway@may.i.e.
}

\begin{abstract}
Young people with disabilities are often excluded from the labour market. The present paper describes an evaluation of an innovative 2-year pilot initiative called Vocational Opportunities in Training for Employment (VOTE) implemented in Northern Ireland to provide employment training and support for vulnerable young adults with a wide range of disabilities. The principal aims of the study were to assess the impact of the new service in the extent to which: (1) it had created and developed training and employment opportunities for young people; and (2) promoted inclusive working partnerships. Documentary analysis was used as a basis for describing and assessing the project objectives in combination with faceto-face interviews with a small number of key stakeholders. A total of 122 young people participated in the initiative in the pilot period, during which time 160 qualifications were obtained. Key stakeholders expressed positive views about the initiative, and in particular, its therapeutic benefits and the extent of interagency working and shared learning. The VOTE initiative achieved considerable success in enabling a significant proportion of young adults to engage in society by developing social and employment skills, and by improving their employment opportunities and prospects. Factors critical to the continued success of this and similar initiatives include: the close involvement of parents; addressing the concerns of local employers; the sympathetic treatment of workplace issues; and effective partnership working.
\end{abstract}

Keywords: disability, employment, VOTE, young people

Accepted for publication 18 July 2003

\section{Introduction}

Young adults with disabilities are significantly less likely than their non-disabled peers to have paid employment (Hirst \& Baldwin 1994, Nordstrom et al. 1998, Morris 1999). They often have much lower aspirations for employment (Walker 1982, Rojewski 1996) and tend to progress from school to day services instead. However, most existing services fail to provide a sufficiently challenging or focused environment for many young adults with a disability. For example, young people with learning disabilities commonly leave school at 19 years of age and move to full- or part-time attendance at a social education centre or statutory day centre. Whilst some of these young people and their parents may prefer the safety and security of conventional services, and consider them to be entirely appropriate, there are many others whose needs would be better met by gaining employment. However, young people with disabilities often do not possess the skills and qualifications necessary to compete for jobs. Many are unable to access further/higher education and training largely because of the lack of specialised facilities, learning materials, and on-site personal care and support. In addition, relatively few employment projects in the UK target specifically young adults with disabilities (Barnes et al. 1998), most of whom are not eligible to access mainstream employment schemes.

Several researchers have noted a lack of understanding amongst employers and employees of the needs of 
people with disabilities (Hirst 1984, Parker 1984, Griffiths 1994). Arguably, business representatives should be closely involved in projects and schemes aimed at developing appropriate employment opportunities for young people with disabilities (McLauglin et al. 2000). To this end, the Department for Education and Employment (1999) has devised a good practice guide for Northern Ireland (NI), but this has had little impact to date and there would appear to be considerable scope for promoting inclusive working environments for people with disabilities. This goal assumes an even greater importance in this European Year for People with Disabilities. Ultimately, there is a need both for effective training and supports for young people with disabilities, and for the engagement and appropriate support of employers and the 'world of work'.

The new employment initiative described in the present paper was implemented within NI's unique integrated health and social services system whereby Health and Social Services Trusts, under the direction of four Health and Social Services Boards, deliver acute hospital services and community health and social services. The four Health and Social Services Boards have responsibilities similar to those held in England and Wales by area health and district health authorities, social services departments and family health services authorities combined. The most significant financial support for those who employ people with disabilities in NI is provided by the Training and Employment Agency (TEA), the statutory body with responsibility for promoting employment, including the provision of support and assistance for people with disabilities and their employers.

\section{The VOTE initiative}

Vocational Opportunities in Training for Employment (VOTE) was originally a 2-year pilot initiative funded, in part, by the EU 'Youthstart' Employment Programme and implemented in NI in 1998. The overall aim of the initiative - which is currently coordinated and partly funded by several local statutory and voluntary organisations - is to provide employment training and support for vulnerable young adults (aged 1825 years) with a wide range of disabilities. Its specific objectives are to: provide work preparation and training; inform and educate employers and parents; and to support participants and employers in work placements and employment.

Four VOTE projects were set up in Belfast and surrounding areas aimed at young people with a wide range of disabilities including learning disabilities, mental health problems and physical disabilities. Each of the four projects is managed by a project group, one of whose members is also part of a larger consortium comprising representatives of the NI funding organisations and principal partners. In turn, this consortium forms part of a transnational partnership known as the Education, Vocational training, and Employment (E.V.E.) Partnership. This involves participants in Austria, Germany, Italy and the Republic of Ireland where comparable programmes of education and employment training for disadvantaged young people have been developed under the auspices of EU Youthstart.

Project 1 is based at an institute of further and higher education, and is aimed primarily at young adults with learning disabilities, but also includes people with a range of physical, sensory and behavioural disabilities. It is managed and funded by Action Mental Health, the largest voluntary sector provider of vocational training services in NI (mainly for people with mental health problems). Project 2 is led by a local voluntary organisation (Mencap) and is located on the premises of a special school for young adults with learning disabilities leaving that school. Project 3 is managed by another voluntary organisation (the Cedar Foundation) and provides support for participants with severe physical disabilities to attend a local college of further and higher education. Project 4 is led by a local Health and Social Services Trust in collaboration with a religious charity. It is aimed at young adults who have been 'looked after' by statutory service providers [under provisions of the Children (NI) Order 1995], although it also includes other young people with similar emotional and behavioural needs. Many of these care leavers tend to have limited community or family support, a typically low level of educational attainment, and a significantly increased risk of homelessness, crime, unemployment and teenage pregnancy.

The present paper reports an evaluation of VOTE, the principal aim of which was to assess the impact of the initiative during its first 18 months in terms of the extent to which: (1) each project had achieved its objectives; (2) training and employment opportunities had been created and developed; and (3) successful working partnerships had been implemented.

\section{Subjects and methods}

Forty-three key documents produced throughout the course of the VOTE pilot period were read and analysed qualitatively by the research team with a view to producing an in-depth, synthesised and independent account of the four VOTE projects. It was not the aim of this study to present the contents of each individual document, but rather, to consider and examine them as an integrated whole in order to assess the overall impact of the initiative. Thus, key messages, themes and findings 
were extracted from documents (using colour coding), and used as a basis for overall description and critical analysis. The documentary material included: funding proposals; narrative reports on the overall initiative; separate project evaluation reports; and qualitative and quantitative research reports pertaining to each of the four projects. For example, research conducted by VOTE staff included a series of surveys undertaken to explore: employers' attitudes to school leavers with complex multiple disabilities; the attitudes of tutors toward young people with disabilities; and trainees' views of VOTE.

This documentary analysis was supplemented by one-to-one interviews conducted by the research team with key stakeholders including project staff, project managers and other members of the NI consortium. A 39-item semi-structured interview called the Evaluating VOTE Interview (EVI) was devised (and completed by the researcher) to obtain a range of information from interviewees on, for example: the operational working and management of each project; the impact of VOTE on local service provision and delivery; perceived benefits and drawbacks; relationships with local employers; the nature and degree of interagency and partnership working; and the extent to which VOTE is characterised by an inclusive approach. This information - collected through a series of open-ended questions - was analysed qualitatively in order to address the three research objectives indicated earlier.

\section{Results}

\section{Administrative and therapeutic outcomes}

The principal administrative or vocational outcomes for the VOTE initiative (Table 1) are based on information obtained mainly from the documentary analysis. The therapeutic benefits of each project, also described below, are based on information obtained from both the documentary analysis and the one-to-one interviews.

\section{Project 1}

Project 1 (institute of further and higher education) was the largest of the four projects, and at the time of the study, involved two full-time support workers, a development officer and a part-time researcher. A range of specialist job skills training courses was provided as well as opportunities for gaining work experience through mainstream, college-based, small business initiatives and other employer-negotiated placements. Fifty-two trainees aged 16-20 years participated in the project, two-thirds of whom had a learning disability. The remainder had a range of disabilities including sensory impairment, mental illness, head injury and stroke. Prospective trainees were referred to the project mainly by the NI TEA, local special schools (or special classes in mainstream schools) and the VOTE development officer. Trainees obtained qualifications in areas such as First Aid, Word Processing and 'Working with Equal People', as well as several NVQ and GCSE qualifications. More than half of the trainees participated in work experience in the retail, catering, motor trade/ transport, manufacturing, and tourism and leisure sectors. Over $80 \%$ of the original placements were sustained whilst several were expanded from the initial one-day-per-week arrangement largely because of the high level of one-to-one induction and support provided by staff. Twenty-two participants did not have a work placement, almost half of whom had not yet completed their training whilst a similar proportion were relatively new 'starts' on the project at the time of the evaluation.

Trainee interviews $(n=29)$ undertaken by VOTE staff indicated positive views about the service and, in particular, a greater sense of community involvement. The responses to the EVI indicated that stakeholders considered the major benefits of the service to be: the high level of one-to-one support; the increase in trainees' confidence; good interagency working; and the relative cost-effectiveness of the service when compared to

Table 1 Key vocational outcomes

\begin{tabular}{|c|c|c|c|c|}
\hline Variable & Project 1 & Project 2 & Project 3 & Project 4 \\
\hline Participants (target number) & $52(45)$ & $13(20)$ & $9(10)$ & $48(16)$ \\
\hline Work experience placements & 38 & 16 & $-{ }^{*}$ & $-^{\star}$ \\
\hline Qualifications gained & 92 (by 37 participants) & 16 (by 8 participants) & 18 (by 9 participants) & 34 (by 15 participants) \\
\hline Into education or training scheme & 26 & 0 & 5 & 5 \\
\hline Into employment & $\begin{array}{l}5 \text { gained work; } \\
1 \text { in voluntary work }\end{array}$ & 1 in voluntary work & $\begin{array}{l}2 \text { gained full-time work; } \\
2 \text { working with } \\
\text { therapeutic earnings }\end{array}$ & $\begin{array}{l}4 \text { full-time; } \\
\text { and } 6 \text { part-time, } \\
\text { with } 11 \text { jobs }\end{array}$ \\
\hline
\end{tabular}

\footnotetext{
* Not part of project.
} 
traditional day care. Networking with employers was also viewed as an essential component of the service, and two postal surveys were completed by project staff to assess needs and disability awareness among 320 employers throughout NI (e.g. 'Employer's attitudes toward disability', VOTE 1999c). The key findings indicated that employers had concerns about possible risks to employees' health and safety, and their own duties under the legislation. Many also had misconceptions about incurring extra costs, and were unaware both of the financial assistance available, and the advice and support on offer from statutory and voluntary organisations. They were also generally in favour of short training courses in disability awareness and ongoing support for disabled employees. These results highlight some important issues when working with employers to secure appropriate employment and placement opportunities for young people with disabilities.

\section{Project 2}

Thirteen young school leavers with learning disabilities, aged between 16 and 20 years, participated in this project. The two staff members, in collaboration with other Mencap staff, developed and implemented a programme of prevocational training called 'Learning for Work'. This includes regular group sessions during which advice about employment and training options is provided, and by which means trainees may discuss their aspirations and expectations of the service and employment issues in general. Project staff also produced a CD-ROM containing readily accessible information on post-school options for young adults with learning disabilities and their parents.

Prior to VOTE, school leavers were on work placement on a one-day-a-week basis for 6-week periods only. However, the VOTE project provided trainees with the opportunity to sample a number and range of work placements over a protracted period of time with dedicated help and support. (Four trainees had sufficiently severe communication, behavioural or health difficulties to be considered unsuitable for work.) It was clear from the information available to the research team that, in the absence of such a service, the teaching staff at the school where the project was based would have had insufficient time over and above their existing workloads to provide intensive one-to-one support, or to liaise with employers in order to source the work placements. The information obtained from the interviews with key stakeholders indicated that there were important therapeutic benefits for trainees in terms of building their confidence and self-esteem, and adding a new dimension to their lives. There were also positive effects for some parents who reported feeling more hopeful and optimistic about their child's future. On a more practical level, the young people were exposed to more realistic and longer-term work placements than they had previously experienced. An employer training and awareness pack was also compiled by VOTE staff following a postal survey of 150 local employers and a series of one-to-one interviews with key statutory and voluntary sector representatives.

\section{Project 3}

This project provided a support service to enable young adults with severe physical difficulties to attend a local college of further and higher education. The project was staffed by one full-time staff member and a pool of support workers. Over 3000 hours of support, including personal care, classroom and study support, were provided to nine participants aged 20-25 years of age (mean $=21$ years), eight of whom obtained qualifications in subjects such as literacy, numeracy, administration and business. The service not only helped the young people to obtain qualifications by accessing mainstream education, but it also helped to reduce the stigma surrounding physical disability by facilitating interaction between the trainees and other students of similar age.

Whilst the average occupancy of nine trainees fell below the target of 10 , the 3082 hours of support exceeded its target by $17 \%$. The total number of support hours increased steadily during the first year of the service and the VOTE project supported 17790 hours of college activity. All of the students who completed an evaluation questionnaire (six out of nine) reported being satisfied with the support that they received, and all felt that the project had helped them to perform better in class and to interact socially with other students. Furthermore, all of the college tutors $(n=14)$ reported that VOTE was either an 'essential' or 'useful' service, although some expressed concerns about the availability of support outside the classroom. Although the arrangement of work placements was not an objective of this project, two participants gained full-time employment at the end of their study period. A further two participants went on to work placements (on therapeutic earnings) whilst three people continued in either full- or part-time education.

\section{Project 4}

The final project was designed for young adults up to 25 years of age who had been 'looked after' by the statutory authorities, as well as others with complex emotional and behavioural needs. The project, staffed by one project officer, recruited 48 participants primarily 
through the social work child-care teams in the Health and Social Services Trust and through the Belfast Mission voluntary organisation that provided premises for the project. Trainees received individual counselling and a series of group sessions on work-related and life-skill topics such as job searching, interview skills and budgeting. Some trainees also received one-to-one advice, guidance and training in preparation for job interviews. These in-house courses appeared to provide a useful springboard for the young people by both helping them to develop a trusting and supportive relationship with project staff, and in equipping them to deal with the world of work. Responses to the EVI suggested that these working relationships were central to the success of the project in engaging the trainees and encouraging full participation. Financial assistance and guidance were also provided to support some trainees to attend courses at further and higher education colleges.

Provision of work placements was not part of this project. However, trainees were matched to potential employment possibilities, and the project worker provided support to these fledgling arrangements. At the end of the study period, four of the young adults had gained full-time employment and six had secured parttime employment, primarily in the fields of retail, catering, manufacturing and social care. Five participants went into full-time education and three had entered 'New Deal' employment schemes. Four had contact with the criminal justice system, and therefore, were unable to take up jobs identified for them.

Information obtained from the EVI indicated that staff had received very positive feedback from the trainees. The positive relationships which developed between the project development officer and the trainees were considered to be a particularly important aspect of the service. By contrast, social workers are often perceived as authority figures, thereby hindering their capacity to engage these typically non-compliant young people. Increased self-esteem among trainees was also noted as an important benefit of the new service. Overall, the information obtained from the face-toface interviews indicated that this project appeared to successfully re-engage most of these vulnerable young people - often viewed as 'no hopers' - by helping them to become increasingly confident and independent, and by enhancing their opportunities of gaining employment through training and development.

\section{Principal common themes/lessons}

According to the information obtained during the evaluation, many of the trainees in the four VOTE projects clearly had the potential, with suitable support, to develop, learn and gain qualifications which they would not otherwise have done. Many also showed that they could perform successfully in a variety of placements and employment settings when given suitable support. The key themes and lessons are outlined below.

\section{Parental involvement}

From January to May 1999, VOTE staff ran a series of focus groups for parents of children and young people involved in some of the projects. They also conducted one-to-one interviews with 15 parents in project 2 in order to ascertain their knowledge and needs. The findings, which are outlined in a number of separate research reports (VOTE 1999a,b), illustrate the importance of maintaining regular contact (where possible) with the families of trainees. In some projects, this was frequent and effective, whilst others had more formal and limited contact with parents. Furthermore, although parents usually attended interviews and review meetings where appropriate, the need for their more active involvement in the referral process was identified by key stakeholders as an area requiring more attention and effort. One potential barrier to parental involvement in the employment futures of their children appeared to be the parents' utilisation of the state benefits system. In line with previous research (e.g. Hirst \& Baldwin 1994), this indicates that parents are concerned about the likelihood of withdrawal of benefits in the event of their child gaining employment. For example, one parent in project 2 appeared to be unhappy about her child undertaking a low-skilled, paid job despite the possible therapeutic benefits. Some parents may also be sometimes reluctant to use the benefits given to them for their child with a disability for that child's benefit. Therefore, there would appear to be a need to address the challenge of trying to overcome perverse incentives in the benefits system and overly protective parental attitudes.

Honesty, sensitivity and realism were all required when dealing with parents because of the potential risk that the VOTE service might give rise to false expectations regarding progress and employment. However, the Code of Practice (Department of Education for Northern Ireland 1998) for school leavers with disability requires that relevant agencies assess parental expectations regarding all aspects of the child's life as part of a transition plan. Despite this, both parents and VOTE staff were uncertain about the links between these legislative requirements and organisational 'on the ground' procedures.

\section{Referrals and referral agents}

Trainees were referred to each project from a wide range of sources. In general, the referral procedures appeared to work well, as evidenced by the sustained 
number of referrals throughout the pilot period. This appears to be largely because of the VOTE partnership arrangements which brought together a range of interested organisations and experienced individuals who were managers of (or at least familiar with) the range of local services. The analysis of relevant VOTE documentation suggested that a series of public relations activities had also contributed significantly to raising awareness of the new initiative. Further information from the EVI indicated that important lessons in this respect were to seek good initial information from referral agents, to be clear about roles, and to maintain good liaison throughout the project.

\section{Employer awareness and workplace issues}

The VOTE staff conducted several surveys aimed at ascertaining employer attitudes towards young people with disabilities (VOTE 1999c). The collective findings indicated that the energy invested in building relationships with, and addressing the concerns of, local employers and their employees is essential to making the transition to 'real' work. Many opportunities for work also depend on matching the person with disabilities with a particular working environment as well as a suitable job opportunity. It is often the non-disabled colleagues who provide a positive or negative working experience as much as the physical aspects or the type of work involved (Barnes et al. 1998). The information on projects 1 and 2, in particular, highlights the importance of offering a variety of placements and workplace supports. Overall, the present findings concur with other research that suggests that there are two main areas which ought to be addressed simultaneously if initiatives such as VOTE are to be fully effective: (1) the needs, attitudes and competencies of potential employees; and (2) the routines and culture of the world of work. Thus, projects need to achieve a good balance and integration between developing participants' readiness for work and addressing the concerns of potential employers, as well as providing appropriate workplace support.

\section{Interagency working}

A final key theme emanating from the VOTE initiative included the need to nurture and sustain successful relationships between staff and trainees and, in particular, to build effective partnership working between diverse organisations. Relationships between staff and trainees appeared to be consistently good within each of the projects and the various sources of evidence combine to suggest that interagency working at local level was generally successful. The local consortium - which meets quarterly in order to share ideas and discuss problems - provides an important mechanism for facilitating interagency working. The VOTE initiative has involved a high level of partnership working between organisations with different cultures and styles of communicating and exercising their power and responsibilities. There is little precedent for statutory agencies (with mandated powers and responsibilities) and voluntary organisations (with perhaps more freedom of decision) working together at the level of partnership seen in the VOTE initiative, and indeed, for these different parts of large organisations working directly together. For example, when organisations are accustomed to acting relatively autonomously, they may view the need to consult with others as a hindrance and/or a loss of efficiency (Gray 1985). One of the key issues learned in terms of the type of partnership working seen here has been the degree of independence and interdependence expected.

\section{Discussion}

It was not the aim of this evaluation to compare the four projects, each of which is unique in terms of target users, specific objectives, methods and resourcing. Therefore, the study findings are discussed collectively with reference to the extent to which the aims of the VOTE initiative had been achieved. In particular, this focuses on the creation of appropriate training and employment opportunities, and the development of successful working partnerships.

\section{The transition to 'real' employment}

The VOTE initiative piloted a range of supports and services designed to enable young adults with disabilities and complex needs to participate in training and employment opportunities. The figures from the four projects are small but encouraging. The capacity of projects 1 and 2 to provide work preparation coupled with a range of supports during placement and in early employment (at relatively short notice and by people known to the trainee) appeared to be a major strength. This supports the findings of a recent systematic review which concluded that supported employment is more effective than prevocational training alone in helping people with severe mental health problems to obtain work (Crowther et al. 2001).

The challenge facing VOTE and similar initiatives is to make a real impact in terms of young people with disabilities entering employment. Given the general employment context in NI and the perverse incentives of the benefits system, it is perhaps unrealistic to expect dramatic improvements in the numbers entering the world of work. A recent study suggests that, although many young people have positive experiences of work placements and job training, few make the actual 
transition to 'real' employment (Monteith 2000). A similar picture emerges from the local TEA employment schemes on which people with a disability are proportionately better represented than in applications for jobs (Gallagher et al. 1994). The close involvement of employers in helping to develop appropriate employment opportunities for young people with disabilities is clearly a critical issue. The VOTE objective of informing and educating employers is perhaps the most challenging in terms of the extent to which it is likely to create real work opportunities for young people with disabilities. The projects which generated employment and placement opportunities (i.e. projects 1 and 2) provided the most concrete evidence of progress in this area. However, less tangible aspects, such as raising employer awareness and changing workplace culture, are more difficult to measure directly, and it is less clear as to whether or not the VOTE initiative has been successful in this respect to date.

It may not be so much opposition as a lack of employer awareness that often impedes employment opportunities for young people with disabilities, many of which need to be matched to individual need and ability. Building trust with local employers and facilitating skills acquisition are as important when facilitating entry to employment as the more tangible vocational outcomes. However, the provision and retention of work placement opportunities is a medium- to longterm goal that may involve intensive levels of support, particularly for young people with learning disabilities. This has obvious resource implications for any future funding of the service which may, however, be offset by the alternative cost of a long-term placement in a local training centre. In general, it is important that each project is able to offer a range of work options and placements, with varying degrees of support. As increasing numbers of young people undertake placements and enter employment, there may be merit in employing a more systematic approach to evaluating job satisfaction (e.g. Nisbet \& York 1989), successful placement (e.g. Dunham et al. 1999) and models of support (e.g. Parsons et al. 2001). This may, in turn, provide useful insights for the selection, matching and support of placements and work opportunities.

\section{A transitional service}

The transition to adulthood is both 'a phase in time and a process of personal growth and development' (McGinty \& Fish 1992). The VOTE initiative seeks to address the transition to employment for young people with disabilities through personal development and by engaging the world of work. The initiative is characterised by an inclusive approach in so far as some partici- pants were accessing mainstream college courses and were beginning to enter the 'real' world of work. An important point to be considered in transitional services of this kind is the nature of the social interaction in working environments. In the world of work, there is likely to be more communication with non-disabled coworkers and with the general public, but perhaps less general social interaction overall than in traditional day-care services (Kilsby \& Beyer 1996) or in educational establishments. Thus, the challenge is to prepare trainees for new types of social environment. Managing changes in social relationships is one of the key tasks of the transition into adulthood, and it merits further attention in future services of this type. A further important aspect of VOTE and similar services is that they are seen as a stepping stone rather than a permanent feature of life. There is evidence to indicate that this may help trainees to reframe their view of the world and their disability as part of the mental preparation for working life (e.g. Gerber et al. 1996). A major challenge is how to ensure a throughput on such schemes while simultaneously providing for each trainee's unique progression.

\section{Partnership between organisations}

The transnational working with other European partners was a recurring theme throughout much of the VOTE documentation and interviews. For example, the VOTE initiative has involved full partnering with joint action plans, commitment of resources and agreed, shared monitoring arrangements. This kind of partnership working was viewed as instrumental in creating a milestone for collective learning and in generating innovative responses to service problems and gaps. The 'pan-disability' nature of this partnership appeared to be a particularly important impetus towards learning about, and creating working relationships across, traditional client group areas. This helps to circumvent the impairment-specific and duplicative nature of many projects highlighted in previous research (Joseph Rowntree Foundation 1998a). The information on which this evaluation was based suggests that the development of successful working partnerships is a key driver in developing specialised services for young people with disabilities, and at least in part, contributes towards the aim of integrating previously excluded young people into mainstream education, training and employment services.

\section{Conclusion}

Overall, the VOTE initiative achieved considerable success during its first 2 years in terms of improving the employment prospects of young adults with a wide range of disabilities and complex needs. Following the 
present authors' pilot evaluation, three out of the four projects received continued funding, whilst the fourth continued in a slightly different form under the auspices of its parent organisation. The various sources of data converge to indicate that the new initiative was, and continues to be, valued highly by service users, providers and commissioners. In particular, the projects appear to have contributed to helping young people who are in a transitional phase of their lives to develop a sense of confidence and self-worth - essential prerequisites for successful participation in training and employment. Although the overall proportion of participants gaining employment may appear low, experience suggests that it is much higher than would have been the case without this initiative. Furthermore, stakeholders indicated that much has been learned about a wide range of issues including: achieving a balance between working with potential employers and working with the world of work; the culture of a service that is transitional; and the challenges and benefits of the inter-organisational partnership working that is necessary to facilitate such ventures.

Each project has been successful in different ways and the collective findings highlight important lessons for the development of similar services elsewhere. These include: (1) identifying appropriate training, placement and employment opportunities (and matching candidates to these on the basis of needs, abilities and aspirations); (2) supporting employers to adapt working practices and workplace 'culture'; (3) establishing successful relationships with parents and referral agents; and (4) implementing the support mechanisms required by young adults with disabilities and complex needs to make the important step from training to paid employment. Arguably, however, what needs to happen once pilot projects such as VOTE have proved their effectiveness, is an integration of specialised employment training and support with mainstream employment or training services (e.g. Gallagher et al. 1994). This should take place mindful of the long and often complex path necessary for most young people with disabilities and complex needs to enter fully the world of work.

\section{Acknowledgements}

We would like to thank the project participants and all of the VOTE staff including, in particular, the project manager, Mrs Linda Leonard.

\section{References}

Barnes H., Thornton P. \& Maynard Campbell S. (1998) Disabled People and Employment: A Review of Research and Development Work. Policy Press, Bristol.
Crowther R.E., Marshall M., Bond G.R. \& Huxley P. (2001) Helping people with severe mental illness to obtain work: systematic review. British Medical Journal 322, 204-208.

Department for Education and Employment (1999) Employing Disabled People - A Good Practice Guide for Managers and Employers. The Stationery Office, London.

Department of Education for Northern Ireland (1998) Code of Practice on the Identification and Assessment of Special Educational Needs Department of Education for Northern Ireland, Belfast.

Dunham M.D., Kulton K.D. \& Koller J.R. (1999) A comparison of adult learning disability subtypes in the vocational rehabilitation system. Rehabilitation Psychology 44, 248-265.

Gallagher A.M., Osborne R.D. \& Rodgers P. (1994) Training and employment. In: Standing Advisory Commission on Human Rights (Ed.) Disability: An Assessment of the Implications of Physical and Sensory Disability in a Northern Ireland Context Together with Supporting Research Papers. HMSO, London.

Gerber P.J., Reiff H.B. \& Ginsberg R. (1996) Reframing the learning disabilities experience. Journal of Learning Disabilities 29, 98-101.

Gray B. (1985) Conditions facilitating inter-organisational collaboration. Human Relations 38, 911-936.

Griffiths M. (1994) Transition to Adulthood - The Role of Education for Young People with Severe Learning Difficulties. David Fulton Publishers, London.

Hirst M.A. (1984) Moving on: Transfer of Young People with Disabilities to Adult Services. Social Policy Research Unit, University of York, York.

Hirst M. \& Baldwin S. (1994) Unequal Opportunities: Growing Up Disabled. HMSO, London.

Joseph Rowntree Foundation (1998a) Findings: Disabled People and Employment: New Issues for Research and Practice. Joseph Rowntree Foundation, York.

Kilsby M. \& Beyer S. (1996) Engagement and interaction: a comparison between supported employment and ATCs. Journal of Community and Applied Social Psychology 6, 141152.

McGinty J. \& Fish J. (1992) Learning Support for Young People: Transition: Leaving School for Further Education and Work. Open University Press, Buckingham.

McLauglin J., Monteith M. \& Sneddon H. (2000) A long and winding road to employment? Young people with disabilities in Northern Ireland: the transition to adulthood. Irish Journal of Social Studies 2, 5-22.

Monteith M. (2000) Making progress? The transition to adulthood for disabled young people in Northern Ireland. In: D. Iwaniec \& M. Hill (Eds) Child Welfare Policy and PracticeIssues and Lessons Emerging from Current Research. Jessica Kingsley Publishers, London.

Morris J. (1999) Move on Up. Barnardo's, Essex.

Nisbet J. \& York P. (1989) Indices of job satisfaction of persons with moderate and severe disabilities. Education and Training in Mental Retardation and Developmental Disabilities 24, 274-280.

Nordstrom C.R., Huffaker B.J. \& Williams K.B. (1998) When physical disabilities are not liabilities: the role of applicant and interviewer characteristics on employment interview outcomes. Journal of Applied Social Psychology 28, 283-306.

Parker G. (1984) Into Work: A Review of the Literature about Disabled Young Adults' Preparation for and Movement into Employment. Social Policy Research Unit, University of York, York. 
Parsons M.B., Reid D.H., Green C.W. \& Browning L.B. (2001) Reducing job coach assistance for supported workers with severe multiple disabilities: an alternative off-site/on-site model. Research in Developmental Disabilities 21, 151-164.

Rojewski J.W. (1996) Occupational aspirations and early career-choice patterns of adolescents with and without learning disabilities. Learning Disability Quarterly 9, 99-116. VOTE (1999a) Project Parents Focus Group 1. VOTE, Craigavon and Banbridge.
VOTE (1999b) Project Parents Focus Group 2. VOTE, Craigavon and Banbridge.

VOTE (1999c) Employer's Attitudes Toward Disability: Report Comparing the Attitudes of Employers in Large and Small Business Communities Toward Disability. VOTE, Craigavon and Banbridge.

Walker A. (1982) Unqualified and Unemployed: Handicapped Young People and the Labour Market. MacMillan Press, London. 